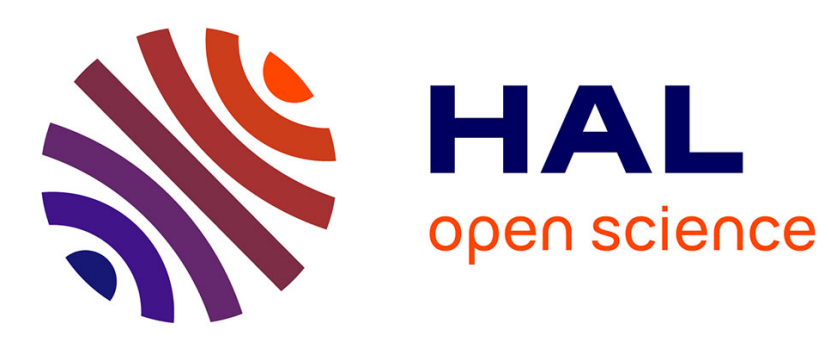

\title{
Thermodynamics based stabilitization of CSTR networks
} Ha Hoang, Françoise Couenne, Yann Le Gorrec, Denis Dochain

\section{To cite this version:}

Ha Hoang, Françoise Couenne, Yann Le Gorrec, Denis Dochain. Thermodynamics based stabilitization of CSTR networks. 51st IEEE Conference on Decision and Control, CDC'12., Dec 2012, Maui, Hawaii, United States. pp.1-8. hal-00803637

\section{HAL Id: hal-00803637 https://hal.science/hal-00803637}

Submitted on 22 Mar 2013

HAL is a multi-disciplinary open access archive for the deposit and dissemination of scientific research documents, whether they are published or not. The documents may come from teaching and research institutions in France or abroad, or from public or private research centers.
L'archive ouverte pluridisciplinaire HAL, est destinée au dépôt et à la diffusion de documents scientifiques de niveau recherche, publiés ou non, émanant des établissements d'enseignement et de recherche français ou étrangers, des laboratoires publics ou privés. 


\title{
Thermodynamics based stabilitization of CSTR networks
}

\author{
H. Hoang, F. Couenne, Y. Le Gorrec and D. Dochain
}

\begin{abstract}
This paper shows that any potential function fulfilling certain thermodynamic stability criteria can be used as a storage function for the Port based modeling of the non isothermal Continuous Stirred Tank Reactor (CSTR) model using the Brayton-Moser formulation without any restriction on the chemical reaction kinetics. By means of an extended IDA-PBC (Interconnection and Damping Assignment-Passivity Based Control) approach, the closed loop energy is then shaped to be equal to the thermal part of the availability with the heat flowrate as the only control input. Some numerical simulations illustrate the theoretical developments.
\end{abstract}

Keywords. Irreversible thermodynamics, CSTR networks, Port Hamiltonian systems, Brayton-Moser, IDA-PBC.

\section{INTRODUCTION}

This paper deals with the control design of continuous single phase chemical reactors in Port Hamiltonian framework using irreversible thermodynamics. More precisely, the Port (pseudo) Hamiltonian model is obtained by using BraytonMoser formulation on the basis of a structured representation of the system with the variables directly issued from thermodynamic considerations. It is shown that there exists some general thermodynamic stability criteria (including the irreversible entropy production due to chemical reaction) that allows to find the (strictly) negative definite matrix $Q$ for the Brayton-Moser formulation. In other words, any thermodynamic variable fulfilling this criteria is usable as an Hamiltonian storage function. This Port Hamiltonian representation can then be used for the control design of the CSTR networks via the IDA-PBC approach [2][8][10][19][21] using the thermodynamic availability function [22][26] as the desired closed loop Hamiltonian.

Let us note that in contrast with mechanical and electrical systems where connections between energy and control are today fairly well understood [12][24], links between physics and chemical process control in a Port Hamiltonian based view are quite difficult to exhibit from a geometrical viewpoint [9]. Indeed, the chemical reaction systems, and in particular the reference case study known as the Continuous Stirred Tank Reactor (CSTR), belong to highly nonlinear non-equilibrium thermodynamic systems via reaction kinetics and irreversibilities of the coupling between matter and

The work of H. Hoang has been funded by VNU-HCM City research project (Vietnam).

H. Hoang is with the Faculty of Chemical Engineering, Ho Chi Minh City University of Technology, 268 Ly Thuong Kiet Str., Dist. 10, HCM City, Vietnam ha. hoangehcmut. edu. vn

F. Couenne is with the LAGEP, University Lyon 1, France

Y. Le Gorrec is with the ENSMM, Besançon, France

D. Dochain is with the CESAME, Université Catholique de Louvain, 1348 Louvain-la-Neuve, Belgium denis.dochain@uclouvain.be temperature. Following the law of conservation of energy, the total energy (the energies of the simple system under consideration and its surrounding medium) is conserved. Consequently the internal energy, that is considered from a microscopic point of view, as the sum of kinetic and potential energies of all molecules inside the system, is then not dissipated during chemical reaction but is modified by exchanges (material and heat flows as well as volume expansion for gas phase reactions) affected at the system boundary as stated by the first principle of thermodynamics. As a consequence, the variation of the internal energy is only to change molecular arrangements and chemical structure of species. Indeed the internal energy cannot be considered as an Hamiltonian function because it does not allow to express the inherent irreversibility of the system governed by the second principle of thermodynamics. The links between thermodynamics and system theory have to be characterized more precisely in order to exhibit thermodynamic variables usable for Port Hamiltonian based control design.

This subject has been an active research area over the years [8][9][10][13][14][16][17][20][21], and the Port Hamiltonian based methodology has been successfully applied in the field of chemical engineering. Even if such a formulation does not formally exist for chemical reaction systems [9], a Port pseudo ${ }^{1}$ Hamiltonian formulation allows to find appropriate feedback laws using the passivity based control [3][10][21].

In this paper, we first show that a passive Port (pseudo) Hamiltonian representation for CSTRs can be derived from the Brayton-Moser formulation when a positivity condition (called thermodynamic stability condition) is satisfied by some chosen potential function. In other words, this formulation is based on a structured representation with the variables directly issued from thermodynamic considerations. In that sense, the paper generalizes the obtained results of [14][20]. We show that the following candidate variables can be chosen as Hamiltonian storage functions :

- the opposite of entropy $-S$ (extensive variable);

- the square of the chemical affinity $\mathscr{A}$ (intensive variable).

In both cases, the dissipation term can be linked to the natural irreversibility (entropy production) due to chemical reaction. Secondly we show that the matching equation of IDA-PBC approach for controlling the CSTR can be easily solved as soon as the desired closed loop Hamiltonian is chosen to be proportional only to the thermal part of the thermodynamic availability function [14][26].

This paper is organized as follows. Section II gives an

${ }^{1}$ Some structure matrices $J(x)$ and $R(x)$ depend also of the co-state variables $\nabla_{x} \mathcal{H}(x)$. 
overview on the links between the Brayton-Moser and Port controlled Hamiltonian representations. Section III presents the CSTR network case study. In the section IV, some preliminary results about a (general) thermodynamic stability criterion and the decomposition of the availability into the thermal and material parts of an ideal multi-component mixture are developed. This stability criteria is then used to find the solution of the Brayton-Moser formulation for the Port (pseudo) Hamiltonian based CSTR modeling with one reaction $\nu_{A} A \rightleftharpoons \nu_{B} B$ in Section V. The IDA-PBC approach is then solved using the thermal part of the availability as the desired close loop Hamiltonian function and the jacket temperature as the only control input. Section VI proposes some numerical simulations.

\section{Port CONTROLled Hamiltonian RePresentation AND BRAYTON-MOSER FORMULATION}

Let us consider open chemical systems that are affine in the control input $u$ and whose dynamics is given by :

$$
\frac{d x}{d t}=f(x)+g(x) u
$$

where $x \in \mathbb{R}^{n}$ is the state vector, $f(x) \in \mathbb{R}^{n}$ represents the smooth nonlinear function with respect to $x, g(x) \in \mathbb{R}^{n \times m}$ is the input-state map and $u \in \mathbb{R}^{m}$ is the input.

\section{A. Brayton-Moser formulation}

The Brayton-Moser formulation [4] requires :

- to find a non singular matrix $Q(x): \mathbb{R}^{n} \rightarrow \mathbb{R}^{n \times n}$ such that its symmetric part is negative definite:

$$
Q(x)+Q(x)^{t} \leq 0
$$

where the exponent $t$ stands for the matrix transpose.

- to write the system dynamics (1) into the following equivalent form :

$$
Q(x) \frac{d x}{d t}=\nabla_{x} P(x)+G(x) u
$$

with $P(x): \mathbb{R}^{n} \rightarrow \mathbb{R}$ is smooth potential function.

From (1)(3) we get the following relations :

$$
\begin{aligned}
\nabla_{x} P(x) & =Q(x) f(x) \\
G(x) & =Q(x) g(x)
\end{aligned}
$$

The sufficient and necessary conditions for the existence of (3) are the symmetry of the Hessian matrix of $P(x)$ :

$$
\mathbb{H}(P)=(\mathbb{H}(P))^{t}
$$

\section{B. Links with the Port controlled Hamiltonian systems}

Because $Q$ is invertible, the Brayton Moser form (3) can be rewritten as follows :

$$
\frac{d x}{d t}=Q(x)^{-1} \nabla_{x} P(x)+g(x) u
$$

Since any square matrix can be split into two (symmetric and skew-symmetric) parts, (7) can be transformed into the following form :

$$
\frac{d x}{d t}=\left[\frac{Q^{-1}-Q^{-1 t}}{2}+\frac{Q^{-1}+Q^{-1 t}}{2}\right] \nabla_{x} P(x)+g(x) u
$$

The above equation can be identified to a general class of the so called Port controlled Hamiltonian ( $\mathrm{PCH}$ ) systems with dissipation given by [12][5][24][25] :

$$
\left\{\begin{array}{l}
\frac{d x}{d t}=[J(x)-R(x)] \nabla_{x} \mathcal{H}(x)+g(x) u \\
y=g(x)^{t} \nabla_{x} \mathcal{H}(x)
\end{array}\right.
$$

with :

$$
\begin{aligned}
\mathcal{H}(x) & =P(x), J(x)=\frac{Q(x)^{-1}-Q(x)^{-1 t}}{2} \\
R(x) & =-\frac{Q(x)^{-1}+Q(x)^{-1 t}}{2}
\end{aligned}
$$

The smooth function $\mathcal{H}(x)=P(x): \mathbb{R}^{n} \rightarrow \mathbb{R}$ represents the Hamiltonian storage function (or the energy) ; $J(x)=$ $-J(x)^{t}$ and $R(x)=R(x)^{t} \geq 0$ are the structure matrices and correspond to the natural interconnection matrix and the damping matrix, respectively; $u, y \in \mathbb{R}^{m}$ are the control input and output, respectively, and are power conjugated port variables. The energy balance immediately follows from (9) :

$$
\frac{d \mathcal{H}(x)}{d t}=-\left[\frac{\partial \mathcal{H}(x)}{\partial x}\right]^{t} R(x)\left[\frac{\partial \mathcal{H}(x)}{\partial x}\right]+u^{t} y
$$

The system (9) is passive in the sense that the dissipation

$$
d=-\left[\frac{\partial \mathcal{H}(x)}{\partial x}\right]^{t} R(x)\left[\frac{\partial \mathcal{H}(x)}{\partial x}\right]<0
$$

is always negative and the Hamiltonian $\mathcal{H}(x)$ is bounded from below [5][25]. The term $d$ defined by (11) corresponds to natural dissipation (energy lost due to friction/damping in mechanical systems or due to resistance in $R L C$ electrical systems [12][24] or due to entropy production in the CSTR networks [14] for example). The following inequality immediately follows from (10)(11) :

$$
\overbrace{\mathcal{H}\left(x\left(t_{2}\right)\right)-\mathcal{H}\left(x\left(t_{1}\right)\right)}^{\text {energy storage }} \leq \overbrace{\int_{t_{1}}^{t_{2}} u(\tau)^{t} y(\tau) d \tau}^{\text {energy supply }}
$$

(12) shows that a passive system cannot store more energy than the one supplied from the environment due to the dissipated energy. With a feedback law of the form $u=-K y$ with $K>0$ this system remains dissipative. In this paper the synthesized feedback laws are based on these Hamiltonian models (9) using Interconnection and Damping Assignment Passivity - Based Control (IDA-PBC) approach [19].

\section{IDA-PBC approach}

The objective of the IDA-PBC approach is to find a static state-feedback control $u=\beta(x)$ such that the closed loop dynamics is also a PCH system with dissipation of the form:

$$
\frac{d x}{d t}=\left[J_{d}(x)-R_{d}(x)\right] \nabla_{x} \mathcal{H}_{d}(x)
$$


where the controlled storage function $\mathcal{H}_{d}(x)$ has a strict local minimum at the desired equilibrium $x^{\star}$, and $J_{d}(x)=$ $-J_{d}(x)^{t}$ and $R_{d}(x)=R_{d}(x)^{t} \geq 0$ are some desired interconnection and damping matrices respectively. The following matching equation that follows immediately from (9)(13) has to be solved :

$$
[J(x)-R(x)] \nabla_{x} \mathcal{H}(x)+g(x) \beta(x)=\left[J_{d}(x)-R_{d}(x)\right] \nabla_{x} \mathcal{H}_{d}(x)
$$

Let consider there exists a full rank left annihilator of $g(x)$ denoted $g(x)^{\perp}$ such that $g(x)^{\perp} g(x)=0$. If $J_{d}(x), R_{d}(x)$ and $\mathcal{H}_{d}(x)$ are chosen such that :

$$
g(x)^{\perp}[J(x)-R(x)] \nabla_{x} \mathcal{H}(x)=g(x)^{\perp}\left[J_{d}(x)-R_{d}(x)\right] \nabla_{x} \mathcal{H}_{d}(x)
$$

the state feedback $\beta(x)$ for $u$ is then given by :

$$
\begin{aligned}
\beta(x)= & \left(g(x)^{t} g(x)\right)^{-1} g(x)^{t}\left(\left[J_{d}(x)-R_{d}(x)\right] \nabla_{x} \mathcal{H}_{d}(x)\right. \\
& \left.-[J(x)-R(x)] \nabla_{x} \mathcal{H}(x)\right)
\end{aligned}
$$

The system (9) with the feedback law (16) is stable at $x^{\star}$ since from (13) we have :

$$
\frac{d \mathcal{H}_{d}(x)}{d t}=-\left(\nabla_{x} \mathcal{H}_{d}(x)\right)^{t} R_{d}(x)\left(\nabla_{x} \mathcal{H}_{d}(x)\right) \leq 0
$$

and $\mathcal{H}_{d}(x)$ is a Lyapunov function. Details on IDA-PBC design procedure can be found in [19].

This paper extends the IDA-PBC approach to the Hamiltonian systems (9) in which the input vector $u=\left(\begin{array}{ll}u_{f} & u_{c}\end{array}\right)^{t}$ contains some input with fixed value $u_{f}$. The stabilization problem is then solved with the only controllable input $u_{c}$.

\section{THE CSTR NETWORKS CASE STUDY}

\section{A. CSTR model}

Let us consider a CSTR with one reaction involving $n$ chemical species $\mathcal{C}_{k}(k=1 \ldots n)$ :

$$
\cdots+\nu_{i} \mathcal{C}_{i}+\cdots=\cdots+\nu_{j} \mathcal{C}_{j}+\cdots
$$

Note that the reaction under consideration can be either a reversible reaction (" = " is then replaced by " $\rightleftharpoons ")$ or an irreversible one (with " = "replaced by " $\rightarrow$ "). $\nu_{i}$ and $\nu_{j}$ are the suitable signed stoichiometric coefficients with the following convention : $\nu_{i}<0$ (resp. $>0$ ) if $\mathcal{C}_{i}$ appears on the left (resp. right) side of (17). Note also that the net reaction rate $r$ is equal to $r=\left(r_{f}-r_{r}\right)$ where $r_{f}$ and $r_{r}$ are the forward and backward reaction rates, respectively.

Let us consider the following assumptions :

(H1) The fluid mixture is ideal, incompressible and under isobaric conditions.

(H2) In the inlet, the reactor is fed by the species $\mathcal{C}_{k}(k=$ $1 \ldots n)$ at a fixed temperature $T_{I}$.

(H3) The heat flow coming from the jacket $\dot{Q}_{J}$

$$
\dot{Q}_{J}=\lambda\left(T_{J}-T\right)
$$

with $\lambda$ the heat exchange coefficient and inlet molar flow rates $\left(F_{1 I}, \ldots, F_{n I}\right)$ are used as inputs.

\section{B. Thermodynamics based view for CSTR modeling}

In thermodynamics, the system variables are divided into extensive variables (such as the internal energy $U$, the entropy $S$, the volume $V$, the molar number $N_{i}$ ) and into intensive variables (such as the temperature $T$, the pressure $P$, the chemical potential $\mu_{i}$ ). In isobaric conditions, the variation of internal energy $U$ is equal to that of the enthalpy $H$, given by considering the Gibbs equation :

$$
d H=\sum_{k=1}^{n} \mu_{k} d N_{k}+T d S
$$

From (19) we have :

$$
d S=\sum_{k=1}^{n} \frac{-\mu_{k}}{T} d N_{k}+\frac{1}{T} d H
$$

Since the entropy $S$ is also an extensive variable, we get :

$$
S\left(N_{1}, \ldots, N_{n}, H\right)=\sum_{k=1}^{n} \frac{-\mu_{k}}{T} N_{k}+\frac{1}{T} H
$$

(20) can be written in a compact form as follows:

$$
d S=w^{t} d Z \quad \Rightarrow \quad w(Z)^{t}=\frac{\partial S(Z)}{\partial Z}
$$

with

$$
w(Z)=\left(\frac{-\mu_{1}}{T}, \ldots, \frac{-\mu_{n}}{T}, \frac{1}{T}\right)^{t}, \quad Z=\left(N_{1}, \ldots, N_{n}, H\right)^{t}
$$

The dynamics of the system is then given by considering the energy and material balances :

$$
\left\{\begin{array}{l}
\frac{d N_{1}}{d t}=F_{1 I}-F_{1}+\nu_{1} r \\
\vdots \\
\frac{d N_{n}}{d t}=F_{n I}-F_{n}+\nu_{n} r \\
\frac{d H}{d t}=\dot{Q}_{J}+\sum_{i=1}^{n}\left(F_{i I} h_{i I}-F_{i} h_{i}\right)
\end{array}\right.
$$

where $\left(F_{1 I}, \ldots, F_{n I}\right), \quad\left(F_{1}, \ldots, F_{n}\right), \quad\left(\nu_{1}, \ldots, \nu_{n}\right)$, $\left(h_{1 I}, \ldots, h_{n I}\right)$ and $\left(h_{1}, \ldots, h_{n}\right)$ and $\dot{Q}_{J}$ are the inlet flow rate vector, the outlet flow rate vector, the stoichiometric vector, the inlet molar enthalpy vector, the molar enthalpy vector and the heat flux coming from the jacket, respectively.

Using the local equilibrium hypothesis [7], (22) can be written as follows :

$$
\frac{d S}{d t}=w^{t} \frac{d Z}{d t}
$$

Let us introduce the definition of the total chemical affinity :

$$
\mathscr{A}=-\nu_{1} \frac{\mu_{1}}{T}-\nu_{2} \frac{\mu_{2}}{T}-\ldots-\nu_{n} \frac{\mu_{n}}{T}
$$

It is shown that $\mathscr{A}(25)$ is also an intensive variable. Recall that the irreversible entropy productions due to reaction $\Sigma_{s}^{\text {reac. }}$ is equal to [15] :

$$
\Sigma_{s}^{\text {reac. }}=\mathscr{A} r V \geq 0
$$

Note that the inequality in (26) always holds for any reaction kinetic constant. We can see that the affinity $\mathscr{A}$ and $r V$ have the same sign for any evolution; if $\mathscr{A}>0$ (resp. $\mathscr{A}<0$ ) then $r V>0$ (resp. $r V<0$ ) and if $\mathscr{A}=0$ then $r V=0$. In other words, the reaction always evolves in the direction of decreasing affinity. 


\section{Thermodynamic availability}

From the second law of thermodynamics for homogeneous systems, the entropy function $S(Z)$ is necessarily concave with respect to $Z$ [6], and as a consequence, it can be shown [22][26] that the availability function $\mathcal{A}$ :

$$
\mathcal{A}(Z)=S_{2}+w_{2}^{t}\left(Z-Z_{2}\right)-S(Z) \geq 0
$$

is non negative, where $Z_{2}$ is some fixed reference point and $w_{2}$ are intensive variables associated to the extensive variables $Z_{2}$. $\mathcal{A}(Z)$ has some interesting properties that will entail its use as Lyapunov function for control purpose [1][14][16][22].

Because the entropy $S$ is an homogeneous function of degree 1 with respect to $Z$ [6], it is strictly concave if and only if at least one global extensive property (such as volume, total mass, total mole number) has been fixing [18]. As a consequence $\mathcal{A}(Z)$ will be strictly convex. In the remaining of the paper we will suppose that the total mass $m_{t}$ in the reactor is constant.

Finally the availability function (27) can be written [14] as follows :

$$
\mathcal{A}(Z)=-\left(w-w_{2}\right)^{t} Z
$$

\section{PREliminary RESUlts}

\section{A. General thermodynamic stability criteria for CSTR}

In the following we define a thermodynamic stability criterion that can be used as a sufficient condition to derive some Port Hamiltonian models from the Brayton-Moser formulation. We shall see that this criterion is strongly related to the second law of thermodynamics.

Definition 1: The thermodynamic potential $P\left(N_{1}, \ldots\right.$, $\left.N_{n}, H\right)$ satisfies the so called general thermodynamic stability criterion if and only if :

$$
\left(-\nu_{1} \frac{\partial P}{\partial N_{1}}-\nu_{2} \frac{\partial P}{\partial N_{2}}-\ldots-\nu_{n} \frac{\partial P}{\partial N_{n}}\right) r V \geq 0
$$

Let us now show that both the chemical affinity (25) and the ectropy (defined as $-S$ ) satisfy the thermodynamic stability criterion (29). Lemma 1 presents a property related to the chemical affinity, generalizing some results given in [11][15].

Lemma 1: From the second law of thermodynamics, the chemical affinity $\mathscr{A}\left(N_{1}, \ldots, N_{n}, H\right)$ verifies :

$$
-\nu_{1} \frac{\partial \mathscr{A}}{\partial N_{1}}-\nu_{2} \frac{\partial \mathscr{A}}{\partial N_{2}}-\ldots-\nu_{n} \frac{\partial \mathscr{A}}{\partial N_{n}} \geq 0
$$

Proof: From (25) we have :

$$
\frac{\partial \mathscr{A}}{\partial N_{i}}=-\sum_{j=1}^{n} \nu_{j} \frac{\partial \frac{\mu_{j}}{T}}{\partial N_{i}} ; i=1, \ldots, n
$$

Taking into account that $-\frac{\mu_{j}}{T}=\frac{\partial S}{\partial N_{j}}$ (see (22)), we have :

$$
\frac{\partial \mathscr{A}}{\partial N_{i}}=\sum_{j=1}^{n} \nu_{j} \frac{\partial^{2} S}{\partial N_{i} \partial N_{j}} ; i=1, \ldots, n
$$

Multiplying (31) by $\nu_{i}$ and summing for $i=1, \ldots, n$, we have :

$$
\sum_{i=1}^{n} \nu_{i} \frac{\partial \mathscr{A}}{\partial N_{i}}=\sum_{i=1}^{n} \sum_{j=1}^{n} \nu_{i} \nu_{j} \frac{\partial^{2} S}{\partial N_{i} \partial N_{j}}
$$

(32) can be also rewritten in the following quadratic form :

$$
\sum_{i=1}^{n} \nu_{i} \frac{\partial \mathscr{A}}{\partial N_{i}}=\boldsymbol{v}^{t} \mathbb{H}(S) \boldsymbol{v}
$$

where $\boldsymbol{v}=\left(0, \nu_{1}, \ldots, \nu_{n}\right)^{t}$ and $\mathbb{H}(S)$ is the Hessian matrix of the entropy function $S(Z)$. Because $S=S(Z)$ is concave with respect to $Z=\left(N_{1}, \ldots, N_{n}, H\right)[6]: \mathbb{H}(S) \leq 0$. (30) immediately follows.

The following Corollary 1 proposes the explicit form of (30) for an ideal multi-component mixture.

Corollary 1: Under the hypothesis (H1), the explicit form of (30) is :

$$
-\sum_{k=1}^{n} \nu_{k} \frac{\partial \mathscr{A}}{\partial N_{k}}=\frac{R}{N_{t}} \boldsymbol{\nu}^{t} \Xi \boldsymbol{\nu}+\left(\boldsymbol{\nu}^{t} \boldsymbol{h}\right)^{2} \frac{1}{C_{p} T^{2}} \geq 0
$$

where $\boldsymbol{\nu}=\left(\nu_{1}, \ldots, \nu_{n}\right)^{t}, \boldsymbol{h}=\left(h_{1}, \ldots, h_{n}\right)^{t}$ and $\Xi$ is the $n \times n$ positive definite matrix :

$\Xi=\left(\xi_{i j}\right)_{i, j=1, \ldots, n}=\left(\begin{array}{cccc}\frac{N_{t}-N_{1}}{N_{1}} & -1 & & -1 \\ -1 & \frac{N_{t}-N_{2}}{N_{2}} & \ddots & \vdots \\ \vdots & \ddots & \ddots & -1 \\ -1 & \ldots & -1 & \frac{N_{t}-N_{n}}{N_{n}}\end{array}\right)$

where $C_{p}=\sum_{i=1}^{n} c_{p i} N_{i}$ is total heat capacity and $N_{t}=$ $\sum_{i=1}^{n} N_{i}$ is total molar number.

Proof: Let us consider an ideal isobaric mixture. The partial molar enthalpies and entropies of the chemical species $k$ are then given by the following expressions [23] :

$$
\left\{\begin{array}{l}
h_{k}\left(T, x_{k}\right)=c_{p k}\left(T-T_{\text {ref }}\right)+h_{k r e f} \\
s_{k}\left(T, x_{k}\right)=c_{p k} \ln \left(\frac{T}{T_{r e f}}\right)+s_{k r e f}-R \ln x_{k}
\end{array}\right.
$$

where $x_{k}$ is molar fraction of species $k(k=1,2, \ldots, n)$ :

$$
x_{k}=\frac{N_{k}}{N_{1}+N_{2}+\ldots+N_{n}}
$$

From $\frac{\mu_{k}}{T}=\frac{h_{k}}{T}-s_{k}$ [7][23], we have :

$$
\left[\frac{\mu_{k}}{T}\right]\left(T, x_{k}\right)=\zeta_{k}(T)+R \ln x_{k}
$$

with

$$
\zeta_{k}(T)=c_{p k}+\frac{-c_{p k} T_{r e f}+h_{k r e f}}{T}-c_{p k} \ln \left(\frac{T}{T_{r e f}}\right)-s_{k r e f}
$$

The total enthalpy can be written as follows :

$$
H\left(T, N_{1}, N_{2}, \ldots, N_{n}\right)=\sum_{k=1}^{n} h_{k} N_{k}
$$

Using the first relation in (36), (40) leads to :

$$
T=T\left(H, N_{1}, \ldots, N_{n}\right)=\frac{H-\sum_{k=1}^{n} N_{k} h_{k r e f}}{\sum_{k=1}^{n} N_{k} c_{p k}}+T_{r e f}
$$

We obtain for $\mathscr{A}(25)$ :

$$
\begin{aligned}
\nu_{i} \frac{\partial \mathscr{A}}{\partial N_{i}} & =-\nu_{i} \nu_{1} \frac{\partial \frac{\mu_{1}}{T}}{\partial N_{i}}-\ldots-\nu_{i} \nu_{n} \frac{\partial \frac{\mu_{n}}{T}}{\partial N_{i}} \\
& =-\nu_{i} \sum_{j=1}^{n} \nu_{j} \frac{\partial \frac{\mu_{j}}{T}}{\partial N_{i}}
\end{aligned}
$$


From (38)(41) we have :

$$
\begin{aligned}
\nu_{i} \frac{\partial \mathscr{A}}{\partial N_{i}}= & -\nu_{i} \sum_{j=1}^{n} \nu_{j} \frac{\partial \zeta_{j}(T)}{\partial T} \frac{\partial T}{\partial N_{i}} \\
& -\nu_{i} R \sum_{j=1}^{n} \nu_{j} \frac{\partial\left(\ln \left(\frac{N_{j}}{N_{1}+\ldots+N_{n}}\right)\right)}{\partial N_{i}}
\end{aligned}
$$

Note from (39)(41) that $\frac{\partial \zeta_{k}(T)}{\partial T}=-\frac{h_{k}}{T^{2}}, \frac{\partial T}{\partial N_{i}}=-\frac{h_{i}}{C_{p}}$. We also have :

$$
\frac{\partial\left(\ln \left(\frac{N_{j}}{N_{1}+N_{2}+\ldots+N_{n}}\right)\right)}{\partial N_{i}}=\left\{\begin{array}{c}
\frac{N_{t}-N_{i}}{N_{i} N_{t}} \quad \text { if } i=j \\
-\frac{1}{N_{t}} \quad \text { if } i \neq j
\end{array}\right.
$$

We obtain for (42) :

$$
\begin{aligned}
\nu_{i} \frac{\partial \mathscr{A}}{\partial N_{i}}= & -\left(\nu_{1} \frac{h_{1}}{T^{2}}+\nu_{2} \frac{h_{2}}{T^{2}}+\ldots+\nu_{n} \frac{h_{n}}{T^{2}}\right) \nu_{i} \frac{h_{i}}{C_{p}} \\
& -R \nu_{i}\left(-\frac{1}{N_{t}} \sum_{j=1, j \neq i}^{n} \nu_{j}+\nu_{i} \frac{N_{t}-N_{i}}{N_{i} N_{t}}\right)
\end{aligned}
$$

Summing (44) for $i=1,2, \ldots, n$, we have :

$$
\begin{aligned}
& -\sum_{i=1}^{n} \nu_{i} \frac{\partial \mathscr{A}}{\partial N_{i}}=\frac{1}{T^{2} C_{p}}\left(\nu_{1} h_{1}+\nu_{2} h_{2}+\ldots+\nu_{n} h_{n}\right)^{2} \\
& +\frac{R}{N_{t}} \sum_{i=1}^{n} \nu_{i}\left(-\sum_{j=1, j \neq i}^{n} \nu_{j}+\nu_{i} \frac{N_{t}-N_{i}}{N_{i}}\right)
\end{aligned}
$$

(45) leads to (34). Finally the positive definiteness of the matrix $\Xi$ (35) immediately follows since it verifies the following conditions : $\xi_{j j}>0$ and $\xi_{i i} \xi_{k k} \geq \frac{1}{4}\left(\xi_{i k}+\xi_{k i}\right)^{2}, \forall i, \forall j, \forall k$. The later ends the proof.

Proposition 1: For any non isothermal homogeneous liquid-phase reaction, $\frac{\mathscr{A}^{2}}{2}$ and $-S$ satisfy the general thermodynamic stability criterion (29).

Proof: The proof immediately follows (the reader can refer to [15] for the simple case of a binary mixture).

Note that the inequality in (29) always holds for any reaction kinetic constant. We can see that the potential $P$ and $r V$ also have the same sign for any evolution.

\section{B. Decomposition of the availability}

The availability function $\mathcal{A}$ (28) can be explicitly written :

$$
\mathcal{A}=-\sum_{k=1}^{n}\left(\frac{-\mu_{k}}{T}+\frac{\mu_{k 2}}{T_{2}}\right) N_{k}-\left(\frac{1}{T}-\frac{1}{T_{2}}\right) H
$$

The Lemma 2 proposes a separation of the availability $\mathcal{A}$ (46) into two parts: the thermal part $\mathcal{A}_{1}$ and the material part $\mathcal{A}_{2}$.

Lemma 2: In the case of an ideal mixture, the availability function (46) can be written as the sum of two functions $\mathcal{A}_{1}$ and $\mathcal{A}_{2}$ such that :

$$
\left\{\begin{array}{l}
\mathcal{A}_{1}\left(N_{1}, \ldots, N_{n}, H\right)=-\sum_{k=1}^{n} \Gamma_{k}(T) N_{k}-\left(\frac{1}{T}-\frac{1}{T_{2}}\right) H \\
\mathcal{A}_{2}\left(N_{1}, \ldots, N_{n}\right)=-\sum_{k=1}^{n} \Lambda_{k}\left(N_{1}, \ldots, N_{n}\right) N_{k}
\end{array}\right.
$$

where

$$
\begin{aligned}
& \Gamma_{k}(T)=c_{p k} \ln \left(\frac{T}{T_{2}}\right)-\frac{h_{k}}{T}+\frac{h_{k 2}}{T_{2}} ; k=1,2, \ldots, n \\
& \Lambda_{k}\left(N_{1}, \ldots, N_{n}\right)=R \ln \left(\frac{N_{k 2} \sum_{i=1}^{n} N_{i}}{N_{k} \sum_{i=1}^{n} N_{i 2}}\right) ; k=1,2, \ldots, n
\end{aligned}
$$

(i) $\mathcal{A}_{2}\left(N_{1}, \ldots, N_{n}\right)$ has the following properties :
- $\mathcal{A}_{2}$ is an homogeneous function of degree one with respect to $\left(N_{1}, \ldots, N_{n}\right)$. Consequently,

$$
\frac{d \mathcal{A}_{2}}{d t}=-\sum_{k=1}^{n} \Lambda_{k}\left(N_{1}, \ldots, N_{n}\right) \frac{d N_{k}}{d t}
$$

(ii) $\mathcal{A}_{1}\left(N_{1}, \ldots, N_{n}, H\right)$ has the following properties :

- $\mathcal{A}_{1} \geq 0$

- $\mathcal{A}_{1}$ is an homogeneous function of degree one with respect to $\left(N_{1}, \ldots, N_{n}, H\right)$. Consequently,

$$
\frac{d \mathcal{A}_{1}}{d t}=-\sum_{k=1}^{n} \Gamma_{k}(T) \frac{d N_{k}}{d t}-\left(\frac{1}{T}-\frac{1}{T_{2}}\right) \frac{d H}{d t}
$$

Proof: From $\frac{\mu_{k}}{T}=\frac{h_{k}}{T}-s_{k}$ [7][23] and (36), we have :

$$
\left(-\frac{\mu_{k}}{T}+\frac{\mu_{k 2}}{T_{2}}\right)=\Gamma_{k}(T)+\Lambda_{k}\left(N_{1}, \ldots, N_{n}\right)
$$

where $\Gamma_{k}(T)$ and $\Lambda_{k}\left(N_{1}, \ldots, N_{n}\right)$ are given in (48). The availability function $\mathcal{A}$ (46) can be then rewritten:

$$
\begin{aligned}
\mathcal{A}\left(N_{1}, \ldots, N_{n}, H\right)= & \mathcal{A}_{1}\left(N_{1}, \ldots, N_{n}, H\right) \\
& +\mathcal{A}_{2}\left(N_{1}, \ldots, N_{n}\right)
\end{aligned}
$$

We refer the reader to [16] for a complete proof.

Remark 1: $\mathcal{A}_{1}$ and $\mathcal{A}_{2}$ are also strictly convex functions with respect to $Z=\left(N_{1}, N_{2}, \ldots, N_{n}, H\right)$ if one constraint on extensive variable has been fixing. We recall that the total mass $m_{t}$ is assumed to be constant. The availability $\mathcal{A}(Z)$ has been successfully used for Lyapunov based control or closed loop Hamiltonian function of reaction systems [1][16][14][22]. In this paper we show $\mathcal{A}_{1}(Z)$ has also same properties as $\mathcal{A}(Z)$ that will entail its use as Lyapunov function candidate for control in the Port Hamiltonian framework.

\section{MAIN RESULTS}

\section{A. Thermodynamically Hamiltonian based CSTR modeling}

For the sake of illustration, let us consider a CSTR with one exothermic reversible reaction $\nu_{A} A \rightleftharpoons \nu_{B} B$. The total mass $m_{t}$ is assumed to be constant. As a consequence, the outlet molar flow rate can directly be expressed from mole fractions and inlet molar flow rate of the different species involved in the reaction [15]. The non isothermal balance equations are then given by (1) with [15] :

$$
\begin{gathered}
x=\left(\begin{array}{c}
N_{A} \\
N_{B} \\
H
\end{array}\right), u=\left(\begin{array}{c}
F_{A I} \\
F_{B I} \\
\dot{Q}_{J}
\end{array}\right), f(x)=\left(\begin{array}{c}
\nu_{A} r \\
\nu_{B} r \\
0
\end{array}\right) \\
g(x)=\left(\begin{array}{ccc}
\left(1-\frac{N_{A} M_{A}}{m_{t}}\right) & -\frac{N_{A} M_{B}}{m_{t}} & 0 \\
-\frac{N_{B} M_{A}}{m_{t}} & \left(1-\frac{N_{B} M_{B}}{m_{t}}\right) & 0 \\
{\left[h_{A I}-\frac{M_{A} H}{m_{t}}\right]} & {\left[h_{B I}-\frac{M_{B} H}{m_{t}}\right]} & 1
\end{array}\right)
\end{gathered}
$$

It has been shown in [14] that the internal energy of the system can be chosen as an Hamiltonian function. The system is then naturally under the Hamiltonian form without dissipation, showing that internal energy is conserved. However, this formulation does not allow to express the inherent 
irreversibility of the reaction from the thermodynamic viewpoint. As a consequence the entropy representation has to be considered and the state variables are $Z=\left(N_{A}, N_{B}, H\right)^{t}$. In this section we show that the proposed criteria (Proposition 1) can be helpful to obtain a Port Hamiltonian representation with dissipation using the Brayton-Moser formulation for the system (1)(53)(54). We only consider the non isothermal case here (yet the isothermal case is deduced similarly).

Proposition 2: In the entropy representation of the binary system with the above reversible reaction, the thermodynamic potential $P$ given in Proposition 1 reduces to :

$$
\left(\nu_{A} \frac{\partial P}{\partial N_{A}}+\nu_{B} \frac{\partial P}{\partial N_{B}}\right) r V \leq 0
$$

If this potential function $P$ verifies the following condition :

$$
\lim _{r \rightarrow 0} \frac{\left(\nu_{A} \frac{\partial P}{\partial N_{A}}+\nu_{B} \frac{\partial P}{\partial N_{B}}\right)}{r}<\infty
$$

then the dynamics $(1)(53)(54)$ can be represented as a Port (pseudo) Hamiltonian system (9) with $x=\left(N_{A}, N_{B}, H\right)^{t}$. Its Hamiltonian storage function is given by $\mathcal{H}(x)=P(x)$ and the structure matrices are written as follows :

$$
\begin{gathered}
J=\frac{1}{2 \Delta}\left(\begin{array}{ccc}
0 & \frac{(-\gamma-2 c) \beta e^{2}}{4(1-\alpha) b} & -\gamma e\left(\frac{\nu_{A}}{\nu_{B}}\right) \\
\frac{-(-\gamma-2 c) \beta e^{2}}{4(1-\alpha) b} & 0 & e c \\
\gamma e\left(\frac{\nu_{A}}{\nu_{B}}\right) & -e c & 0
\end{array}\right) \\
R=-\frac{1}{2 \Delta}\left(\begin{array}{ccc}
\frac{2 \alpha \beta e^{2}\left(\frac{\nu_{A}}{\nu_{B}}\right)^{2}}{(1-\alpha)} & \frac{\alpha \beta e^{2} \frac{\nu_{A}}{\nu_{B}}}{(1-\alpha)} & \gamma e\left(\frac{\nu_{A}}{\nu_{B}}\right) \\
\frac{\alpha \beta e^{2} \frac{\nu_{A}}{\nu_{B}}}{(1-\alpha)} & \frac{\beta e^{2}}{2(1-\alpha)} & -e c \\
\gamma e\left(\frac{\nu_{A}}{\nu_{B}}\right) & -e c & 2\left[\gamma\left(b\left(\frac{\nu_{A}}{\nu_{B}}\right)+c\right)+c^{2}\right]
\end{array}\right)
\end{gathered}
$$

with $0<\alpha<1, \beta>1$, and

$$
\begin{aligned}
\gamma & =4 \alpha b \frac{\nu_{A}}{\nu_{B}}, b=\frac{1}{\nu_{A}}\left(\frac{\nu_{B}}{\nu_{A}} \frac{\partial P}{\partial N_{B}}+\frac{\partial P}{\partial N_{A}}\right) \frac{1}{r V} \\
c & =-\frac{1}{\nu_{A}} \frac{\partial P}{\partial N_{B}} \frac{1}{r V}, e=-\frac{1}{\nu_{A}}\left(\frac{\partial P}{\partial H} \frac{1}{r V}\right) \\
\Delta & =\frac{\alpha \beta e^{2}}{(1-\alpha)}\left(b\left(\frac{\nu_{A}}{\nu_{B}}\right)^{2}+c \frac{\nu_{A}}{\nu_{B}}\right)+\frac{\beta e^{2} c^{2}}{4(1-\alpha) b}
\end{aligned}
$$

The input-state map is $g(x)$ given by (54), and the input $u$ by (53). The output $y$ is $y=g^{t} \nabla_{x} \mathcal{H}(x)$. Finally, the system is passive with dissipation (11):

$$
d=\left(\nu_{A} \frac{\partial P}{\partial N_{A}}+\nu_{B} \frac{\partial P}{\partial N_{B}}\right) r V
$$

Proof: The proof is done by using the Brayton-Moser Formulation (see Section II). We refer the reader to [15] for more details of the proof and some remarks therein.

Note that the obtained results give a global dissipative behavior for non isothermal case without any variable transformation as in [20].

\section{B. IDA-PBC control}

In this subsection we propose a feedback law to stabilize the system at a desired state $x_{d}=Z_{d}=\left(N_{A d}, N_{B d}, H_{d}\right)^{t}$ using the heat flow coming from the jacket $\dot{Q}_{J}(18)$ as the only input control for practical implementation and the thermal part $\mathcal{A}_{1}$ of the availability (47) as the desired closed loop

Hamiltonian function. This choice induces indeed smoother variations of the control variable with respect to previous results [14]. It is completely stated in Proposition 3.

Proposition 3: The system is globally asymptotically stable and admits $x_{d}=Z_{d}=\left(N_{A d}, N_{B d}, H_{d}\right)^{t} \equiv Z_{2}$ as the desired operating point with the following state feedback :

$$
\begin{aligned}
\dot{Q}_{J}= & -\left[h_{A I}-\frac{M_{A} H}{m_{t}}\right] F_{A I}-\left[h_{B I}-\frac{M_{B} H}{m_{t}}\right] F_{B I} \\
& -K \frac{\partial \mathcal{H}_{d}}{\partial H}-\left(\frac{\partial \mathcal{H}_{d}}{\partial H}\right)^{-1}\left(\frac{d N_{A}}{d t} \frac{\partial \mathcal{H}_{d}}{\partial N_{A}}+\frac{d N_{B}}{d t} \frac{\partial \mathcal{H}_{d}}{\partial N_{B}}\right)
\end{aligned}
$$

And the controlled system is a pseudo Hamiltonian system :

$$
\frac{d x}{d t}=\left[J_{d}(x)-R_{d}(x)\right] \nabla_{x} \mathcal{H}_{d}(x)
$$

with

$$
\mathcal{H}_{d}(x)=\mathcal{A}_{1}(x)
$$

$$
\begin{aligned}
& J_{d}(x)=\left(\begin{array}{cc}
0 & 0 \\
0 & 0 \\
-\left(\frac{\partial \mathscr{H}_{d}}{\partial H}\right)^{-1} \frac{d N_{A}}{d t} & -\left(\frac{\partial \mathscr{H}_{d}}{\partial H}\right)^{-1} \frac{d N_{B}}{d t}
\end{array}\right. \\
& R_{d}(x)=\left(\begin{array}{ccc}
0 & 0 & 0 \\
0 & 0 & 0 \\
0 & 0 & K
\end{array}\right)
\end{aligned}
$$

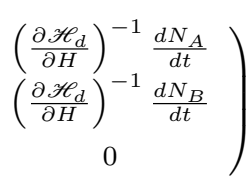

with $K$ a positive tuning parameter.

Proof: Let us consider the dynamics (1)(53)(54) with its Port (pseudo) Hamiltonian representation given by Proposition 2 with only one control input $u=\dot{Q}_{J}$. With $g(x)=$ $\left(g_{1}(x) g_{2}(x) g_{3}(x)\right)$, the Port Hamiltonian model of the system is then rewritten as follows :

$$
\begin{aligned}
\frac{d x}{d t}= & {[J(x)-R(x)] \nabla_{x} \mathcal{H}(x)+\left(g_{1}(x) g_{2}(x)\right)\left(\begin{array}{c}
F_{A I} \\
F_{B I}
\end{array}\right) } \\
& +g_{3} \dot{Q}_{J}
\end{aligned}
$$

Let us consider the IDA-PBC approach (see subsection II-C) and note that

$$
g_{3}(x)^{\perp}=\left(M_{A} M_{B} 0\right)
$$

is a full rank left annihilator of $g_{3}(x)$. It remains to choose a desired closed loop (convex) Hamiltonian function $\mathcal{H}_{d}(x)$ admitting $x_{d}=Z_{d}=\left(N_{A d}, N_{B d}, H_{d}\right)^{t}$ as a minimum, some structure matrices $J_{d}(x)$ and $R_{d}(x)$ and to find $\dot{Q}_{J}$ such that :

$$
\begin{gathered}
{[J(x)-R(x)] \nabla_{x} \mathcal{H}(x)+\left(g_{1}(x) g_{2}(x)\right)\left(\begin{array}{c}
F_{A I} \\
F_{B I}
\end{array}\right)} \\
+g_{3} \dot{Q}_{J}=M_{d}(x) \nabla_{x} \mathcal{H}_{d}(x)
\end{gathered}
$$

where $M_{d}(x)=J_{d}(x)-R_{d}(x)=\left(m_{i j}(x)\right)_{i, j=1,2,3}$. Multiplying (64) by $g_{3}(x)^{\perp}$ from (63), we have :

$$
0=g_{3}^{\perp}(x) M_{d}(x) \nabla_{x} \mathcal{H}_{d}
$$

i.e. :

$$
0=\left(M_{A} M_{B}\right)\left(\begin{array}{lll}
m_{11}(x) & m_{12}(x) & m_{13}(x) \\
m_{21}(x) & m_{22}(x) & m_{23}(x)
\end{array}\right)\left(\begin{array}{c}
\frac{\partial \mathscr{H}_{d}}{\partial N_{A}} \\
\frac{\partial \mathscr{H}_{d}}{\partial N_{B}} \\
\frac{\partial \mathscr{H}_{d}}{\partial H}
\end{array}\right)
$$


With $m_{11}(x)=m_{12}(x)=m_{21}(x)=m_{22}(x)=0, m_{13}(x)$ $=-m_{31}(x)=\left(\frac{\partial \mathscr{H}_{d}}{\partial H}\right)^{-1} \frac{d N_{A}}{d t}, m_{23}(x)=-m_{32}(x)=$ $\left(\frac{\partial \mathscr{H}_{d}}{\partial H}\right)^{-1} \frac{d N_{B}}{d t}$ and $m_{33}(x)=-K<0, M_{d}(x)$ becomes :

$$
\left(\begin{array}{ccc}
0 & 0 & \left(\frac{\partial \mathscr{H}_{d}}{\partial H}\right)^{-1} \frac{d N_{A}}{d t} \\
0 & 0 & \left(\frac{\partial \mathscr{H}_{d}}{\partial H}\right)^{-1} \frac{d N_{B}}{d t} \\
-\left(\frac{\partial \mathscr{H}_{d}}{\partial H}\right)^{-1} \frac{d N_{A}}{d t} & -\left(\frac{\partial \mathscr{H}_{d}}{\partial H}\right)^{-1} \frac{d N_{B}}{d t} & -K
\end{array}\right)
$$

The matrix $M_{d}(x)$ leads to the matrix $J_{d}(x)(61)$ and $R_{d}(x)$ (62). From the matching equation (64) we obtain the control law (58). Finally one can check that $\mathcal{H}_{d}\left(x_{d}\right)=0$ and that the stabilization immediately follows since:

$$
\frac{\partial \mathcal{H}_{d}}{\partial t}=\frac{\partial \mathcal{H}_{d}}{\partial x} \frac{\partial x}{\partial t}=-K\left(\frac{\partial \mathscr{H}_{d}}{\partial H}\right)^{2}<0, \quad \forall x \neq x_{d}
$$

Let us note that

$$
\mathcal{H}_{d}(x)=\mathcal{A}_{1}(x) \Rightarrow\left\{\begin{array}{l}
\frac{\partial \mathcal{H}_{d}}{\partial H}=-\left(\frac{1}{T}-\frac{1}{T_{d}}\right) \\
\frac{\partial \mathcal{H}_{d}}{\partial N_{A}}=-\Gamma_{A}\left(T, T_{d}\right) \\
\frac{\partial \mathcal{H}_{d}}{\partial N_{B}}=-\Gamma_{B}\left(T, T_{d}\right)
\end{array}\right.
$$

$\Gamma_{A}\left(T, T_{d}\right)$ and $\Gamma_{B}\left(T, T_{d}\right)$ are given in (48). $T_{d}$ is the desired temperature at $Z_{d}=\left(N_{A d}, N_{B d}, H_{d}\right)^{t}$. As a consequence, the feedback law (58) is well-defined because $\left(\frac{\partial \mathcal{H}_{d}}{\partial H}\right)^{-1} \frac{\partial \mathcal{H}_{d}}{\partial N_{A}}$ and $\left(\frac{\partial \mathcal{H}_{d}}{\partial H}\right)^{-1} \frac{\partial \mathcal{H}_{d}}{\partial N_{B}}$ are $C^{\infty}$ with respect to $T$.

Remark 2: With the feedback law (58), the dynamics of the enthalpy $H$ becomes :

$\frac{d H}{d t}=-K \frac{\partial \mathcal{H}_{d}}{\partial H}-\left(\frac{\partial \mathcal{H}_{d}}{\partial H}\right)^{-1}\left(\frac{d N_{A}}{d t} \frac{\partial \mathcal{H}_{d}}{\partial N_{A}}+\frac{d N_{B}}{d t} \frac{\partial \mathcal{H}_{d}}{\partial N_{B}}\right)$

Using $H=h_{A} N_{A}+h_{B} N_{B}$ then $\frac{d H}{d t}=C_{p} \frac{d T}{d t}+h_{A} \frac{d N_{A}}{d t}+$ $h_{B} \frac{d N_{B}}{d t}$, and we obtain :

$$
\begin{aligned}
C_{p} \frac{d T}{d t}= & -K \frac{\partial \mathcal{H}_{d}}{\partial H}-\left\{\left[\left(\frac{\partial \mathcal{H}_{d}}{\partial H}\right)^{-1} \frac{\partial \mathcal{H}_{d}}{\partial N_{A}}+h_{A}\right] \frac{d N_{A}}{d t}\right. \\
+ & {\left.\left[\left(\frac{\partial \mathcal{H}_{d}}{\partial H}\right)^{-1} \frac{\partial \mathcal{H}_{d}}{\partial N_{B}}+h_{B}\right] \frac{d N_{B}}{d t}\right\} }
\end{aligned}
$$

At $T=T_{d}, \mathcal{H}_{d}(x)=0$. As a consequence, (67) insures that at the limit $T=T_{d}$ :

$\lim _{T \rightarrow T_{d}}\left(\frac{\partial \mathcal{H}_{d}}{\partial H}\right)^{-1} \frac{d N_{A}}{d t}<\infty, \quad \lim _{T \rightarrow T_{d}}\left(\frac{\partial \mathcal{H}_{d}}{\partial H}\right)^{-1} \frac{d N_{B}}{d t}<\infty$

\section{NUMERICAL SIMULATIONS}

Let us consider first order kinetics with respect to both $A$ and $B$, e.g. $-\nu_{A}=\nu_{B}=1$. Let us also assume that only the component $A$ is fed with molar flow rate $F_{A I}$ at a fixed temperature $T_{I}$, and that the kinetics of the liquid phase reaction is modelled thanks to the Arrhenius law: $k(T)$ $=k_{0} \exp \left(\frac{-E_{a}}{R T}\right)$, i.e. that the net reaction rate is then given by $: r V=k(T)\left(\exp \left(\frac{\mu_{A}}{R T}\right)-\exp \left(\frac{\mu_{B}}{R T}\right)\right)$.

The following parameter values are considered :

$$
\begin{aligned}
c_{p A} & =45.24 \mathrm{~J} / \mathrm{K} / \mathrm{mol}, c_{p B}=30 \mathrm{~J} / \mathrm{K} / \mathrm{mol}, \\
E_{a} & =60.426 \mathrm{KJ} / \mathrm{mol}, h_{\text {Aref }}=0 \mathrm{~J} / \mathrm{mol}, \\
h_{\text {Bref }} & =-9560.6 \mathrm{~J} / \mathrm{mol}, P=10^{5} \mathrm{~Pa}, T_{\text {ref }}=300 \mathrm{~K}
\end{aligned}
$$

Simulations are performed from four initial conditions.

\begin{tabular}{llll}
\hline$\left(C_{1}\right)$ & $T_{0}=350$ & $N_{A 0}=0.7$ & $N_{B 0}=1.3$ \\
$\left(C_{2}\right)$ & $T_{0}=335$ & $N_{A 0}=1.5$ & $N_{B 0}=0.5$ \\
$\left(C_{3}\right)$ & $T_{0}=310$ & $N_{A 0}=1.6$ & $N_{B 0}=0.4$ \\
$\left(C_{4}\right)$ & $T_{0}=300$ & $N_{A 0}=1$ & $N_{B 0}=1$ \\
\hline
\end{tabular}

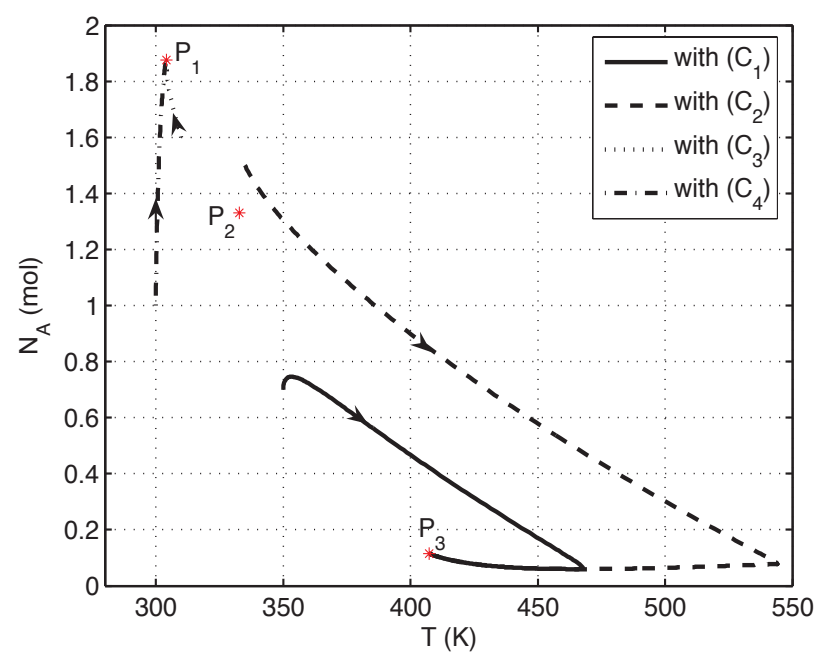

Fig. 1. Some trajectories in the phase plane

Let us consider the following input values in open loop : $F_{A I}=0.0183 \mathrm{~mol} / \mathrm{s}, T_{I}=298 \mathrm{~K}, T_{J}=298 \mathrm{~K}$. The system has then three possible steady states $P_{1}, P_{2}$ and $P_{3}$ [16]. $P_{1}$ and $P_{3}$ are asymptotically stable while $P_{2}$ is instable. Open loop simulations are given in Fig. 1.

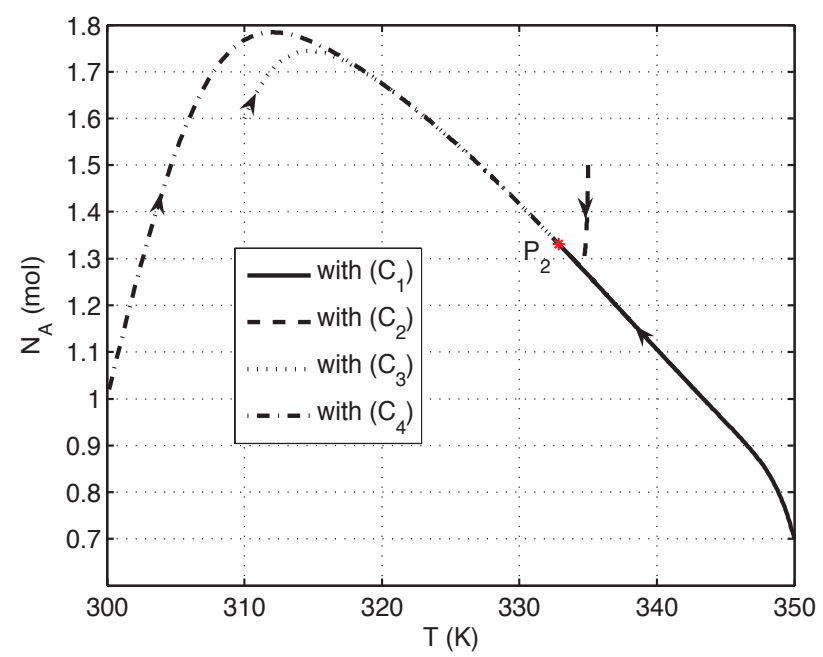

Fig. 2. Phase plane of the controlled non isothermal system

Let us consider the control of the reactor at the unstable steady state operating point $P_{2}$ corresponding to $T=332.85$ $\mathrm{K}$ and at fixed $T_{I}$ and $F_{A I}$ by acting on $T_{J}$. The feedback law for $T_{J}$ is then derived from (18)(58): $T_{J}=\frac{Q_{J}}{\lambda}+T$ (where $\lambda=0.94 \mathrm{~W} / \mathrm{K}$ is used). Fig. 2 gives closed loop simulations 


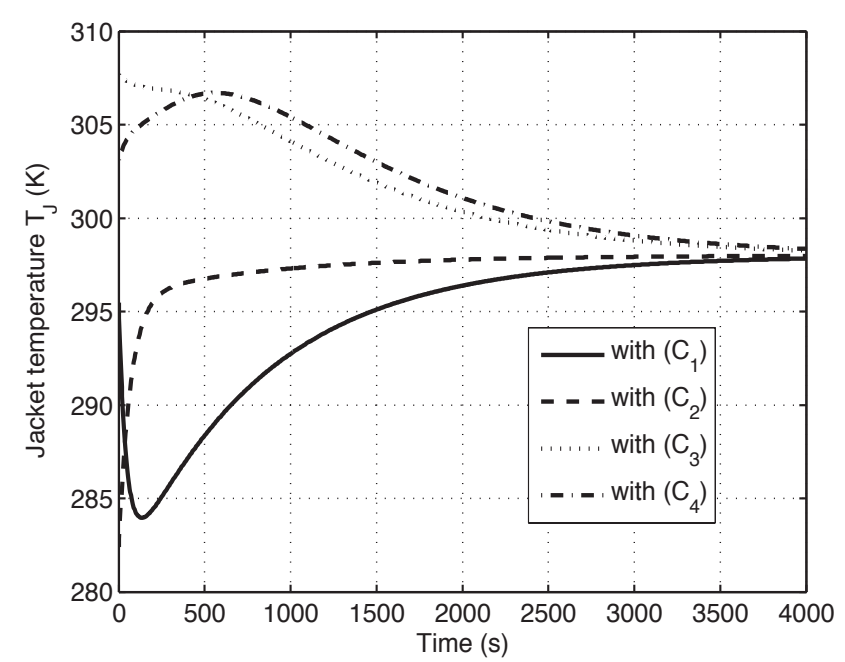

Fig. 3. Dynamics of the control input $T_{J}$

where the tuning parameter $K=10000$. The controlled dynamical trajectories converge to the instable point $P_{2}$ for all considered initial conditions. The controlled input $T_{J}$ is given in Fig. 3. Its dynamics is slow enough, smooth and admissible.

\section{REFERENCES}

[1] Alonso A. A. and B.E. Ydstie (2001). Stabilization of Distributed Systems using Irreversible Thermodynamics, Automatica, $37: 1739-1755$.

[2] Alvarez J., J. Alvarez-Ramírez, G. Espinosa-Perez and A. Schaum (2011). Energy shaping plus damping injection control for a class of chemical reactors. Chem. Eng. Sci., 66(23), 6280-6286.

[3] Bao J. and P. L. Lee (2007). Process control: the passive systems approach. Springer.

[4] Brayton R. K and J. K. Moser (1964). A theory of nonlinear networks I., Quaterly Applied Math., 22:1-33.

[5] Brogliato B., R. Lozano, B. Maschke and O. Egeland (2007). Dissipative systems analysis and control. Springer, London.

[6] Callen H. B. (1985). Thermodynamics and an introduction to thermostatics. John Wiley \& Sons, New York.

[7] De Groot S. R. and P. Mazur (1962). Non-equilibrium thermodynamics. Dover Pub. Inc., Amsterdam.

[8] Dörfler F., J.K. Johnsen and F. Allgöwer (2009). An introduction to interconnection and damping assignment passivity-based control in process engineering. J. Proc. Control, 19, 1413-1426.

[9] Eberard D., B. Maschke and A. van der Schaft (2007). An extension of pseudo-Hamiltonian systems to the thermodynamic space: towards a geometry of nonequilibrium thermodynamics. Rep. Math. Physics, 60(2), 175-198.
[10] Favache A. and D. Dochain (2010). Power-shaping of reaction systems : the CSTR case study. Automatica, 46(11), 1877-1883.

[11] Favache A. D. Dochain and J. Winkin (2011). Powershaping control: writing the system dynamics into the Brayton-Moser form. Syst. Cont. Let., 60(8), 618-624.

[12] Maschke B., R. Ortega and A. van der Schaft (2000). Energy based Lyapunov functions for forced Hamiltonian systems with dissipation. IEEE TAC, 45(8), 14981502.

[13] Hangos K. M., J. Bokor and G. Szederkényi(2001). Hamiltonian view on process systems, AIChE J., 47(8), 1819-1831.

[14] Hoang H., F. Couenne, C. Jallut and Y. Le Gorrec (2011a). The Port Hamiltonian approach to modeling and control of Continuous Stirred Tank Reactors. J. Proc. Control, 21, 1449 - 1458.

[15] Hoang H., F. Couenne, D. Dochain and Y. Le Gorrec (2011b). From Brayton-Moser formulation to Port Hamiltonian representation: the CSTR case study. IFAC World congress, Milano, Italy. 1628-1633.

[16] Hoang H., F. Couenne, C. Jallut and Y. Le Gorrec (2012). Lyapunov-based control of non isothermal continuous stirred tank reactors using irreversible thermodynamics. J. Proc. Control, to appear.

[17] Hudon N., K. Hoffner and M. Guay (2008). Equivalence to dissipative Hamiltonian realization. Proc. 47th IEEE Conference on Decision and Control. 3163-3168.

[18] Jillson K. R. and B.E. Ydstie (2007). Process netwroks with decentralized inventory and flow control. J. Proc. Control, 17, 399-413.

[19] Ortega R., A. van der Schaft, B. Maschke and G. Escobar (2002). Interconnection and damping assignment passivity-based control of port-controlled Hamiltonian Systems. Automatica, 38, 585-596.

[20] Otero-Muras I., G. Szederkényi, A.A. Alonso and K.M. Hangos (2008). Local dissipative Hamiltonian description of reversible reaction networks. Syst. Contr. Let., 57(7), 554-560.

[21] Ramírez H., D. Sbarbaro and R. Ortega(2009). On the control of non-linear processes: An IDA-PBC approach. J. Proc. Control, 19, 405-414.

[22] Ruszkowski M., V. Garcia-Osorio and B.E. Ydstie (2005). Passivity based control of transport reaction systems. AIChE J., 51, 3147-3166.

[23] Sandler S. I. (1999). Chemical and Engineering Thermodynamics. Wiley and Sons, 3rd edition.

[24] van der Schaft A. (2000a). Port-controlled Hamiltonian systems: towards a theory for control and design of nonlinear physical systems. SICE journal, 39(2), 91-98.

[25] van der Schaft A. (2000b). $L_{2}$-gain and passivity techniques in nonlinear control. Springer-Verlag, London.

[26] Ydstie B. E., and A.A. Alonso (1997). Process systems and passivity via the Clausius-Planck inequality. Syst. Cont. Let., 30(5), 253-264. 